\title{
Factors affecting the effective number of tests in genetic association studies: a comparative study of three PCA-based methods
}

\author{
Shu-Hui Wen ${ }^{1}$ and Zhi-Sheng Lu$^{2}$
}

The number of tested marker becomes numerous in genetic association studies (GAS) and one major challenge is to derive the multiple testing threshold. Some approaches calculating an effective number $\left(\boldsymbol{M}_{\mathrm{eff}}\right)$ of tests in GAS were developed and have been shown to be promising. As yet, there have been no comparisons of their robustness to influencing factors. We evaluated the performance of three principal component analysis (PCA)-based $M_{\text {eff }}$ estimation formulas ( $M_{\text {eff-C }}$ in Cheverud (2001), $M_{\text {eff }-\mathrm{L}}$ in $\mathrm{Li}$ and $\mathrm{Ji}$ (2005), and $M_{\mathrm{eff}-\mathrm{G}}$ in Galwey (2009)). Four influencing factors including LD measurements, marker density, population samples and the total number of tested markers were considered. We validated them by the Bonferroni's method and the permutation test with 10000 random shuffles based on three real data sets. For each factor, $M_{\text {eff-C }}$ yielded conservative threshold except with $D^{\prime}$ coefficient, and $M_{\text {eff-G }}$ would be too liberal compared with the permutation test. Our results indicated that $M_{\text {eff-L }}$ based on $r^{2}$ coefficient achieve close approximation of the permutation threshold. As for a large number of markers, we recommended to use $M_{\text {eff-L }}$ with $r^{2}$ coefficient according to fixed-length separation, as well as fixed-number separation, to obtain accurate estimate of the multiple testing threshold and to save more computational time.

Journal of Human Genetics (2011) 56, 428-435; doi:10.1038/jhg.2011.34; published online 31 March 2011

Keywords: correlated multiple testing; effective number; linkage disequilibrium; permutation test; principal component analysis

\section{INTRODUCTION}

Genetic association analysis has been used widely in the search for genes contributing to complex diseases. In addition to the single nucleotide polymorphisms (SNPs) $\mathrm{Map}^{1}$ and the haplotype map (HapMap) projects, ${ }^{2,3}$ several large-scale studies collected genome data from diseased and normal individuals using association tests. ${ }^{4-9}$ By comparing their allele frequencies or marker genotypes such as the SNPs, researchers try to identify candidate regions for further fine mapping. ${ }^{10-12}$ High-throughput genotyping makes genome-wide association studies a realistic mapping strategy, as found in the $500 \mathrm{k}$ or $300 \mathrm{k}$ study. However, analysis of the large-scale data encounters the multiple testing dilemmas that most traditional statistical tests failing in reducing the chances of both false positives and false negatives. ${ }^{10,12-14}$ With numerous SNPs in genome-wide association studies, linkage disequilibrium (LD) ensures that there exists correlation between tests conducted on nearby SNPs. A shortcoming of using traditional methods, such as the Bonferroni's method, is unnecessarily conservative because (1) it fails to account for the dependencies between tests and (2) it involves a large number of tests.

Many strategies for correlated multiple tests in genetic association studies (GAS) have been presented. One standard strategy for estimating the adjusted significance level for correlated multiple testing is the permutation test. ${ }^{15}$ It reserves the correlation among $M$ markers as only the phenotype data are randomly shuffled. However, the accuracy depends on a large number of replications, and it was very time consuming for large $M$. Therefore, another convenient alternative approach is to calculate the effective number $\left(M_{\mathrm{eff}}\right)$. It was an estimate of an 'independent' set of $M$ markers that filtered the correlations among markers from the genotype correlation matrix. The $M_{\text {eff }}$ can be calculated analytically; hence, it is faster and more efficient than the permutation test. Once the number is calculated, it could be used to adjust the significance level as 0.05 divided by the $M_{\text {eff }}$ according to the Bonferroni's correction.

For calculating the effective number, there exists three common formulas based on the principal component analysis (PCA). Cheverud $^{16}$ first proposed performing the PCA on the $M \times M$ Pearson's correlation coefficient matrix. For $M$ eigenvalues (that is, $\lambda_{1}, \ldots, \lambda_{\mathrm{M}}$ ), the variance of $M$ eigenvalues (that is, $V(\lambda)$ ) divided by $M$ represents the strength of dependences. For example, if all markers are completely correlated, the $M$ eigenvalues are: $\lambda_{1}=M, \lambda_{2}=\ldots=\lambda_{\mathrm{M}}=0$, the strength of dependences turns out to be 1 . Therefore, the effective number could be calculated as $M_{\text {eff-C }}=1+(M-1)\left(1-\frac{V(\lambda)}{M}\right)$. But the drawback is that Cheverud used the Pearson's correlation rather

${ }^{1}$ Department of Public Health, College of Medicine, Tzu-Chi University, Hualien, Taiwan and ${ }^{2}$ Community Health Division, Bureau of Health Promotion, Department of Health, New Taipei City, Taiwan

Correspondence: Dr S-H Wen, Department of Public Health, College of Medicine, Tzu-Chi University, Hualien 97004, Taiwan.

E-mail: shwen@mail.tcu.edu.tw

Received 14 December 2010; revised 15 February 2011; accepted 1 March 2011; published online 31 March 2011 
than considering the LD coefficient. Thus, there are some modifications of the measurements of correlations among $M$ SNPs; for example, Nyholt ${ }^{17}$ adopted the LD coefficient (that is, $r$ coefficient) based on the $M_{\text {eff-C }}$ equation. Another formula was defined as $M_{\text {eff }-\mathrm{L}}=\sum_{i=1}^{M} I\left(\lambda_{\mathrm{i}} \geq 1\right)+\left(\lambda_{\mathrm{i}}-\left\lfloor\lambda_{\mathrm{i}}\right\rfloor\right)$ in $\mathrm{Li}$ and $\mathrm{Ji}^{18}{ }^{18}$ where $\mathrm{I}$ is an identity function and $|$.$| denotes a floor function. The first part is$ similar to selecting the principal components according to eigenvaluegreater-than-one rule. ${ }^{19}$ However, the second part might overweight on the decimal part of $\lambda_{\mathrm{i}}$. Hence, Galwey ${ }^{20}$ suggested to weight the eigenvalues directly according to the ratio function as $M_{\text {eff-G }}=\left(\sum_{i=1}^{M} \sqrt{\lambda_{i}}\right)^{2} / \sum_{i=1}^{M} \lambda_{i}$. This formula was strongly related to a moment-based estimator proposed by Patterson et al. ${ }^{21}$ for the purpose of detecting population structure. However, Dudbridge and Gusnanto $^{22}$ had found that Patterson's estimator would be too liberal for deriving the significance threshold of correlated multiple testing.

As yet, there have been no comparisons of their robustness with influencing factors in large-scale GAS. Thus, it is of practical interest to determine whether the estimated effective numbers of tests from the three calculation formulas are different. To address this, three real data sets were used to investigate the performance of the three PCAbased formulas (that is, $M_{\text {eff-C }}, M_{\text {eff-L }}$, and $M_{\text {eff-G }}$ ) for correlated multiple testing. In particular, we examined the impact of four factors including the various measurements of $\mathrm{LD}$, the strength of dependences among $M$ markers, the population samples and the total number of tests on deriving the effective number. We describe in detail comparison analyses of the three formulas, as well as compared them with the Bonferroni's method and the permutation test in terms of the estimated effective number and the adjusted significance level (that is, $\alpha_{\mathrm{p}}$ ).

\section{MATERIALS AND METHODS}

\section{Influencing factors}

To use the correlation among $M$ markers, the measurements of LD have a key role, where LD refers to the correlation among neighboring alleles. We adopt four widely used measures of pairwise LD between all pairs of biallelic loci, including $D^{23}$, Lewontin's standardized disequilibrium coefficient $D^{\prime}, r$ and $r^{2} .^{24,25}$ Suppose that two bi-allelic loci with alleles $\mathrm{A} / \mathrm{a}$ and $\mathrm{B} / \mathrm{b}$ and the corresponding allele frequencies are $\mathrm{P}_{\mathrm{A}}, \mathrm{P}_{\mathrm{a}}, \mathrm{P}_{\mathrm{B}}$ and $\mathrm{P}_{\mathrm{b}}$. The definition of $D$ is $\mathrm{P}_{A B}-\mathrm{P}_{A} \mathrm{P}_{B}$ where $\mathrm{P}_{\mathrm{AB}}$ is the frequency of haplotype $\mathrm{AB}$. Other LD measurements can be viewed as different standardizations of the $D$ coefficient. For example, the $D^{\prime}=|D| / D_{\max }$ and $r=D /\left(P_{A} P_{a} P_{B} P_{b}\right)^{1 / 2}$. SNPs of different minor allele frequencies (MAFs), on average, have different LD properties. ${ }^{25,26}$ Hence, this might lead to a different estimate of the effective number according to the eigenvalues of the PCA based on LD matrix. In addition, the strength of dependencies among tested $M$ SNPs might influence the LD estimates, and we design low and high marker density according to physical distances (in kb) to reflect the different magnitude of overall dependence while controlling the same number of tested SNPs. As several studies ${ }^{3,27}$ have shown differences in LD and haplotype block patterns in populations, we further consider the influence of population samples, such as Asian, European and African population, on the estimated effective number. For the fourth factor, a large number of total SNPs might result in a computation burden in performing the PCA on the genotype correlation matrix. We propose to divide the M SNPs into small blocks and make the calculation of the effective number more flexible.

\section{Test data sets}

For the purpose of comparative analyses of current PCA-based adjustment formulas including: $M_{\text {eff-C }}, M_{\text {eff-L }}$ and $M_{\text {eff-G }}$, we used three real data sets for demonstration. The first data set pertains to the CYP2D6 drug-metabolizing activity. It contains 27 markers over an $880 \mathrm{~kb}$ region with a large sample size. ${ }^{28}$ All 27 SNPs had a MAF of $>5 \%$. We will use these data to demonstrate the impact of LD measurements on calculating the effective number. The second application is a genome-wide association studies about osteonecrosis of the jaw, $^{8}$ and we selected 22144 SNPs with MAFs $>5 \%$ over $135135.6 \mathrm{~kb}$ on the chromosome 10. These data are used to investigate the influence of various $M$ and strength of dependencies. We varied the value of $M$ in a range from 100 to 2000 keeping similar marker density. The numbers of controls for the two data sets are 977 and 65, respectively. The basic characteristics for the two data sets are shown in Supplementary Table S1. The average missing rate ranges from 2.4 to $2.9 \%$. The marker density, defined as the average distance between SNPs, ranges from 4.3 to $67.6 \mathrm{~kb}$. The larger marker density represents that the true overall strength of dependence would be smaller than that with a smaller marker density. As a third example, the SNPs data from the International Haplotype Map (HapMap) Project were used to evaluate the influences of population samples. We examined population differentiation for $2000 \mathrm{com}$ monly shared SNPs with MAFs $>5 \%$ on Chromosome 10 in population samples from the International HapMap Project data (Public release 27, merged II+III).

\section{Calculating the effective number}

The common feature of the three formulas $M_{\text {eff-C }}, M_{\text {eff-L }}$ and $M_{\text {eff-G }}$ is based on the eigenvalues. We implemented the PCA on the $M \times M$ genotype LD matrix between healthy individuals, by using the $D, D^{\prime}, r$ and $r^{2}$ coefficient separately for each of the other influencing factors. Thereafter, the estimated effective number could be obtained according to each formula in each scenario we studied. Negative eigenvalues sometimes occurred and were set to zero in inferring the effective number. In this study, they are relatively few in number and close to zero. We propose using all eigenvalues except negative ones to derive the effective number. It should be noted that some researchers suggested selecting the eigenvalues so that the amount of variance was explained achieving a predefined proportion; for example 99.5\%, as suggested in Gao et al. ${ }^{29}$

Large $M$ might lead to an intensive computational burden in performing the PCA; hence, we also proposed three separation methods for $M$ SNPs into small sets. Partition methods are fixed length, fixed number, and LD block for dividing $M$ consecutive SNPs into segments containing a small number of contiguous loci. Next, we applied the three PCA-based formulas to each subset. The sum of the inferred effective number for each subset was the estimated as the effective number for the entire $M$ SNPs. To evaluate the quality of $M_{\text {eff-C }}, M_{\text {eff-L }}$ and $M_{\text {eff-G }}$ to the permutation test in terms of the adjusted significance level $\alpha_{p}$, we carried out 10000 permutations on our data sets. In each random shuffle, the individuals were randomly assigned as either cases or controls limited to the same disease rate of the first two original case-control data. ${ }^{8,28}$ However, for HapMap data with only healthy individuals, half of the individuals were randomly assigned as cases and the other half were assigned as controls. Keeping the genotypes fixed, the minimum $P$-values in all SNPs obtained from the Fisher's exact test for the permuted case-control samples was recorded in each permutation. The Fisher's exact test was based on comparing allele frequency between cases and controls. The fifth percentile was served as the permutation-based significance threshold (that is, $\alpha_{\mathrm{p}-\text { permut }}$ ) for the overall $5 \%$ type I error rate. We used the statistical software R for the computations. ${ }^{30}$

\section{RESULTS}

\section{The influences of LD coefficients and marker density}

Take the CYP2D6 data (977 controls; 41 cases) for illustration, the range for $D$ coefficient is $(-0.097,0.238),(0.0026,0.9995)$ for $D^{\prime}$ coefficient, $(-0.4805,0.9555)$ for $r$ coefficient, and $(0,0.9131)$ for $r^{2}$ coefficient (Pairwise LD plot was shown in Supplementary Figure S1). The magnitudes of these four LD measurements have different patterns of association with the observed marker alleles. Table 1 lists the estimated effective numbers and significance levels of the tests (that is, $0.05 / M_{\text {eff }}$ ) from three PCA-based formulas according to different values of $M$. The differences among three formulas are very small for $D$ coefficient, followed by $r^{2}$. The estimated effective numbers are obviously different for $D^{\prime}$ and $r$ coefficient, regardless of various values of $M$. Among four LD coefficients, the effective number 
Table 1 Estimated effective numbers and significance levels of tests from three PCA-based formulas using different LD coefficients

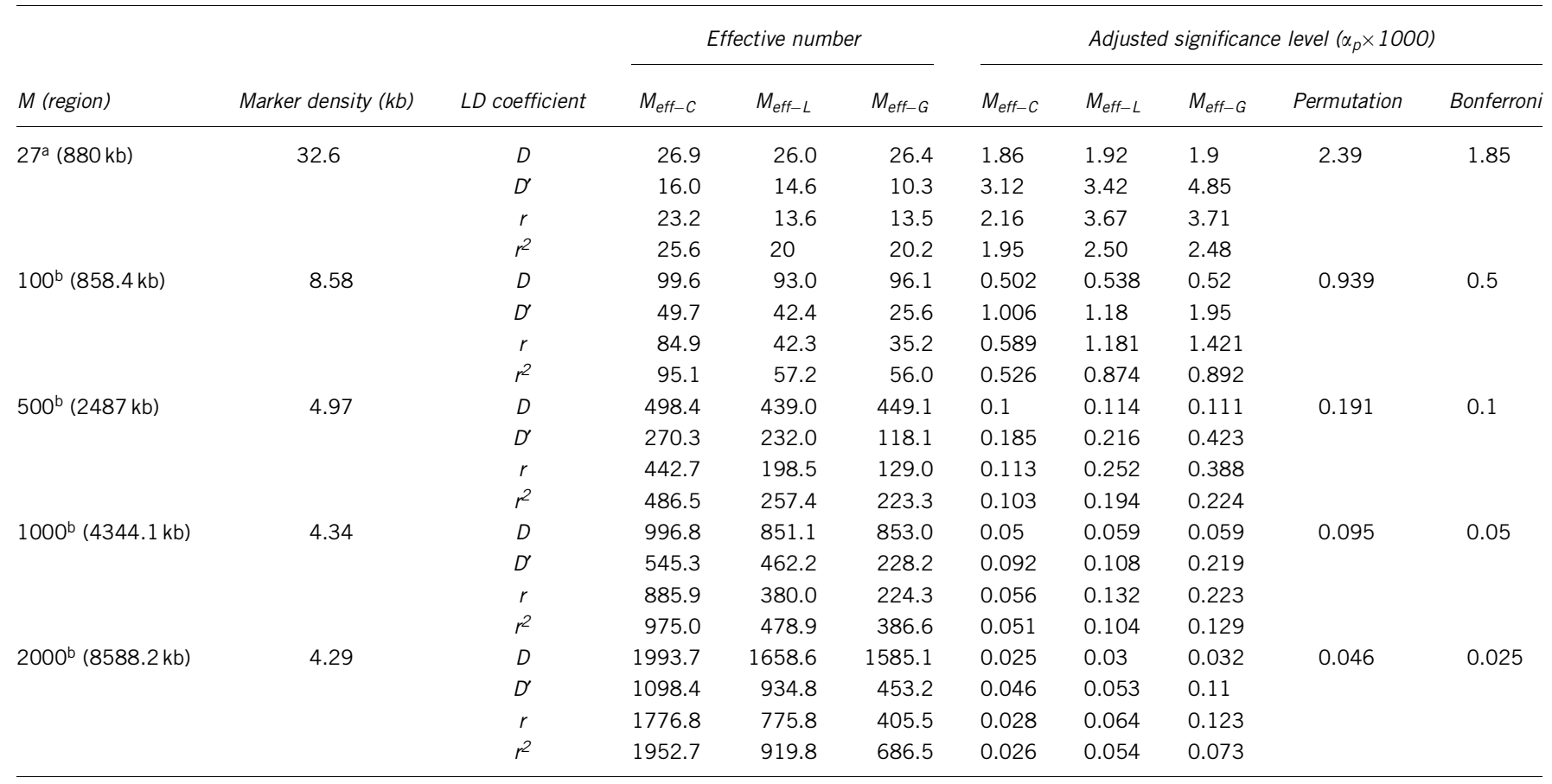

aThe CYP2D6 data from Hosking et al. ${ }^{28}$

bata on chromosome 10 from Sarasquete et al. ${ }^{8}$

a

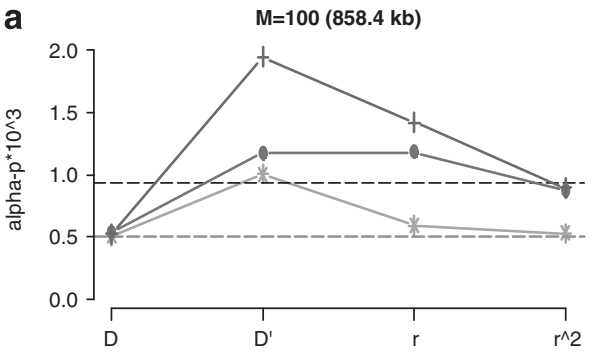

C

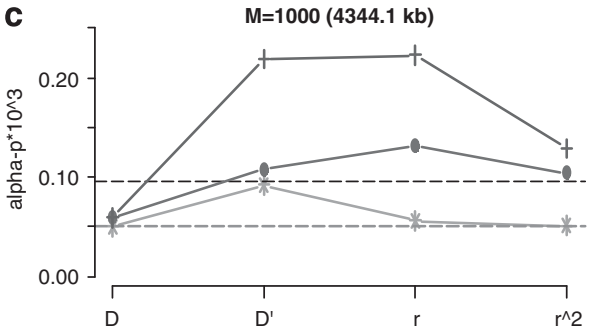

b
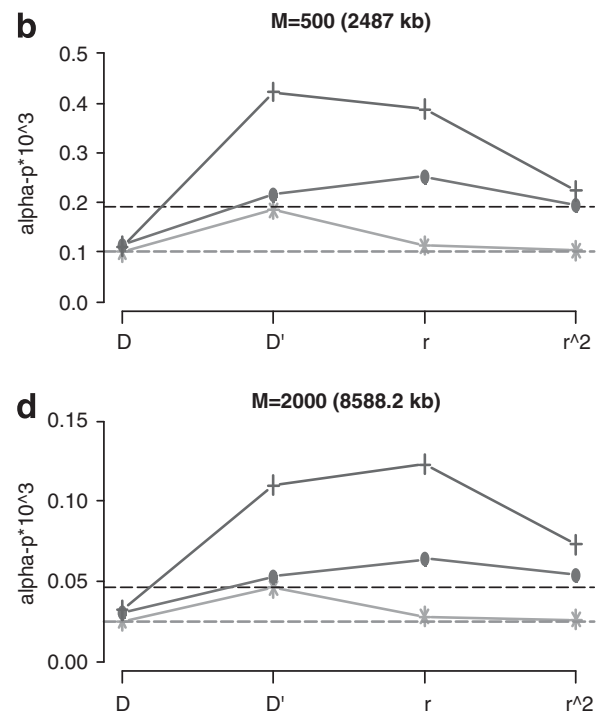

Figure 1 Significance thresholds from three principal component analysis (PCA)-based formulas, the permutation test and Bonferroni's correction at different $M$. (a) $M=100$; (b) $M=500$; (c) $M=1000$; (d) $M=2000$ single nucleotide polymorphisms (SNPs) on chromosome 10 from Sarasquete et al. ${ }^{8}$ Green line denotes the Cheverud's formula, red line denotes $\mathrm{Li}$ and Ji's formula and blue line denotes Galwey's formula. Black dotted line represents the permutation test and pink dotted line denotes Bonferroni's correction. A full color version of this figure is available at the Journal of Human Genetics journal online.

estimated using the $D$ coefficient is the largest and seems to be as stringent as the Bonferroni's method regardless of which formula. Compare with the permutation test, Cheverud's formula $\left(M_{\mathrm{eff}-\mathrm{C}}\right)$ leads to the most conservative results except with $D^{\prime}$ coefficient, followed by $\mathrm{Li}$ and Ji's formula $\left(M_{\text {eff-L }}\right)$, and Galwey's formula $\left(M_{\text {eff-G }}\right)$ is the most liberal, regardless of LD coefficients and various values of $M$.
Figure 1 shows the significance thresholds from three PCA-based methods, the Bonferroni's method and the permutation test at different values of $M(M=100,500,1000,2000$, with similar marker density). Compared with the standard from the permutation test, the threshold from $M_{\text {eff-C }}$ with $D^{\prime}$ coefficient performs closer to the standard at dense marker density (one SNP per $4-9 \mathrm{~kb}$ on average) even when the sample size is small $(n=65)$. As the total number 

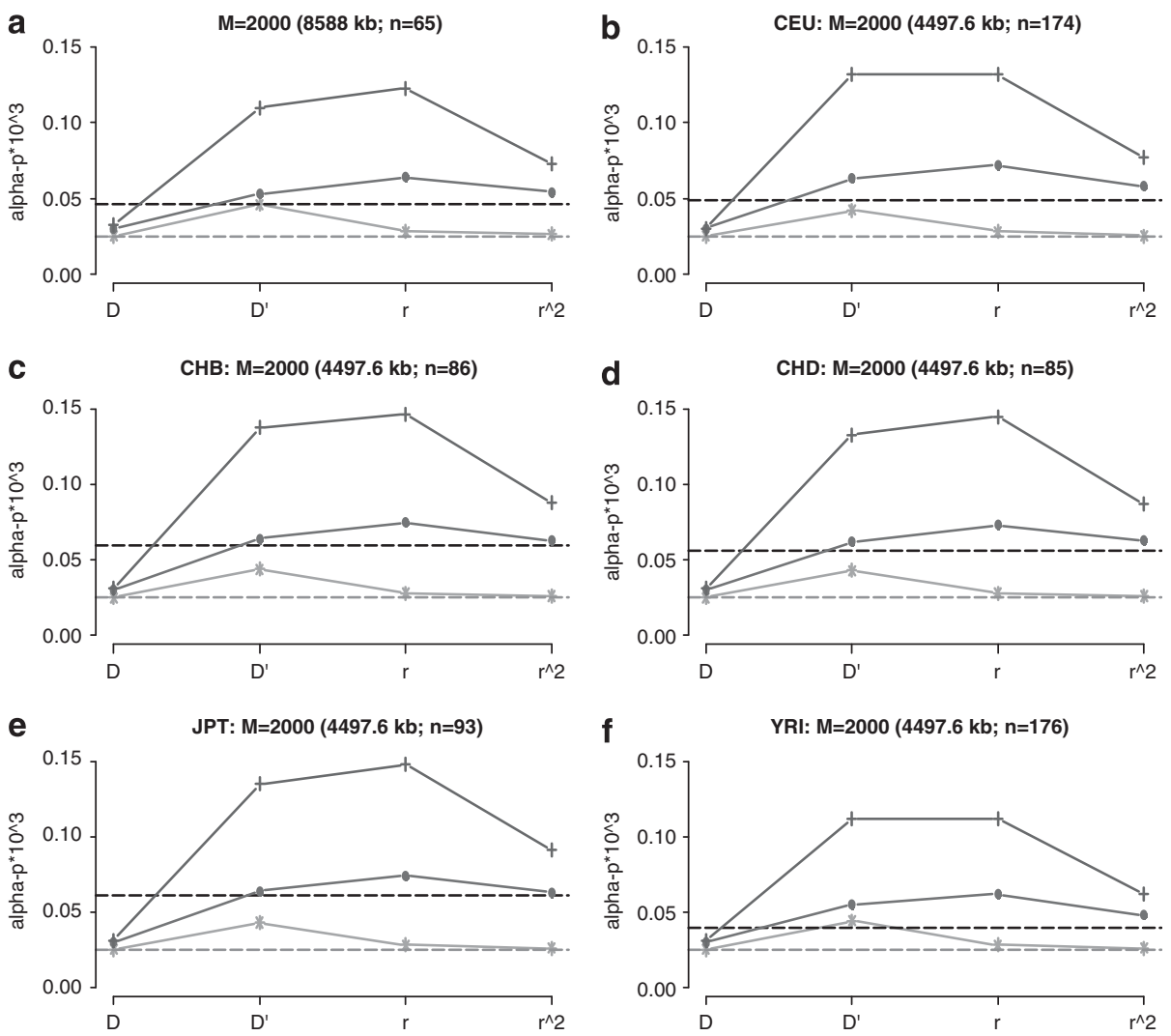

Figure 2 Significance thresholds from three principal component analysis (PCA)-based formulas, the permutation test and Bonferroni's correction in different populations ( $M=2000$ on chromosome 10). (a) Data from Sarasquete et al. ${ }^{8}$; (b) CEU; (c) CHB; (d) CHD; (e) JPT; and (f) YRI from the HapMap data. Green line denotes the Cheverud's formula, red line denotes Li and Ji's formula and blue line denotes Galwey's formula. Black dotted line represents the permutation test and pink dotted line denotes Bonferroni's correction. A full color version of this figure is available at the Journal of Human Genetics journal online.

of tests increases, the estimated thresholds according to $M_{\text {eff-L }}$ with either $D^{\prime}$ or $r^{2}$ coefficient and $M_{\mathrm{eff}-\mathrm{C}}$ with $D^{\prime}$ coefficient are very close to each other, as well as nearly reach the standard. However, the $M_{\text {eff-G }}$ with $r^{2}$ coefficient tends to be slightly liberal. It is worth noting that $M_{\mathrm{eff}-\mathrm{G}}$ with either $D^{\prime}$ or $r$ coefficient results in a very liberal threshold regardless of sample size and the total number of tests. In other words, the overall false-positive rate might be inflated.

Next, to investigate the influences of marker density, the term 'marker density' refers to the strength of association among $M$ SNPs; for example, increasing marker density decreases the strength of association. We further examined the impact of marker density on the estimated effective number for $M_{\text {eff-C }}, M_{\text {eff-L }}$ and $M_{\text {eff-G }}$. Having controlled for $M=2000$, we randomly selected 2000 markers out of 22144 SNPs on chromosome 10, and the average marker density was $67.57 \mathrm{~kb}$ per SNP. The strength of dependence was smaller than the previous 2000 SNPs with an average marker density at $4.29 \mathrm{~kb}$.

Supplementary Figure S2 shows the significance thresholds at different marker density. The permutation test did obtain a larger threshold, as the overall dependence was stronger under the same $M$ $\left(\alpha_{\text {p-permut }}=4.6 \times 10^{-5}\right.$ and $3.7 \times 10^{-5}$ for the smaller and larger marker density, respectively). Although the estimated effective numbers rose as expected for the larger marker density given the same $M$, the growth for $M_{\mathrm{eff}-\mathrm{C}}$ was the lowest. Namely, Cheverud's formula could not adequately reflect the changes in the overall dependence structure. As for $M_{\text {eff-L }}$ and $M_{\text {eff-G }}$, the differences in estimated effective numbers are only obvious with application to $r^{2}$ coefficient $\left(M_{\mathrm{eff}-\mathrm{L}}=919.8\right.$ and 1039.7 for smaller and larger marker density, respectively; $M_{\text {eff-G }}=686.5$ and 784.4 for smaller and larger marker density, respectively). Furthermore, if the strength of dependence is smaller, $M_{\text {eff-C }}$ with $D^{\prime}$ coefficient might overestimate the adjusted significance level. It is found that the most conservative formula $M_{\text {eff-C }}$ would perform better only with $D^{\prime}$ coefficient for data with both a small marker density ( $5 \mathrm{~kb}$ per SNP) and a small sample size.

\section{The influences of the study population}

Since the evolution history of different populations that might result in different magnitudes of $\mathrm{LD}$, we investigated the impact of the study populations from the HapMap data. Five populations including CEU (Northern and Western European; $n=174$ ), CHB (Han Chinese in Beijing, China; $n=86$ ), CHD (Chinese in Metropolitan Denver, Colorado; $n=85$ ), JPT (Japanese in Tokyo, Japan; $n=93$ ) and YRI (Yoruba in Ibadan, Nigeria; $n=176$ ) were used for study. For comparison among five populations, we selected commonly shared SNPs $(M=100,2000)$ with MAF $>5 \%$ located on Chromosome 10. Figure 2 presents the estimated thresholds for five populations (similar results for $M=100$ are shown in Supplementary Figure S3). A very similar pattern for three formulas according to various LD measurements was obtained for each population. The closer the populations, the more similar the patterns of the estimated effective number (or $\left.\alpha_{p}\right)$ profiles. For example, the results of Asian populations such as $\mathrm{CHB}, \mathrm{CHD}$ and JPT were quite similar. For CEU, the results were consistent with those found in data from Sarasquete et al. ${ }^{8}$ however, these two populations were closer. The effective number was relatively large in 

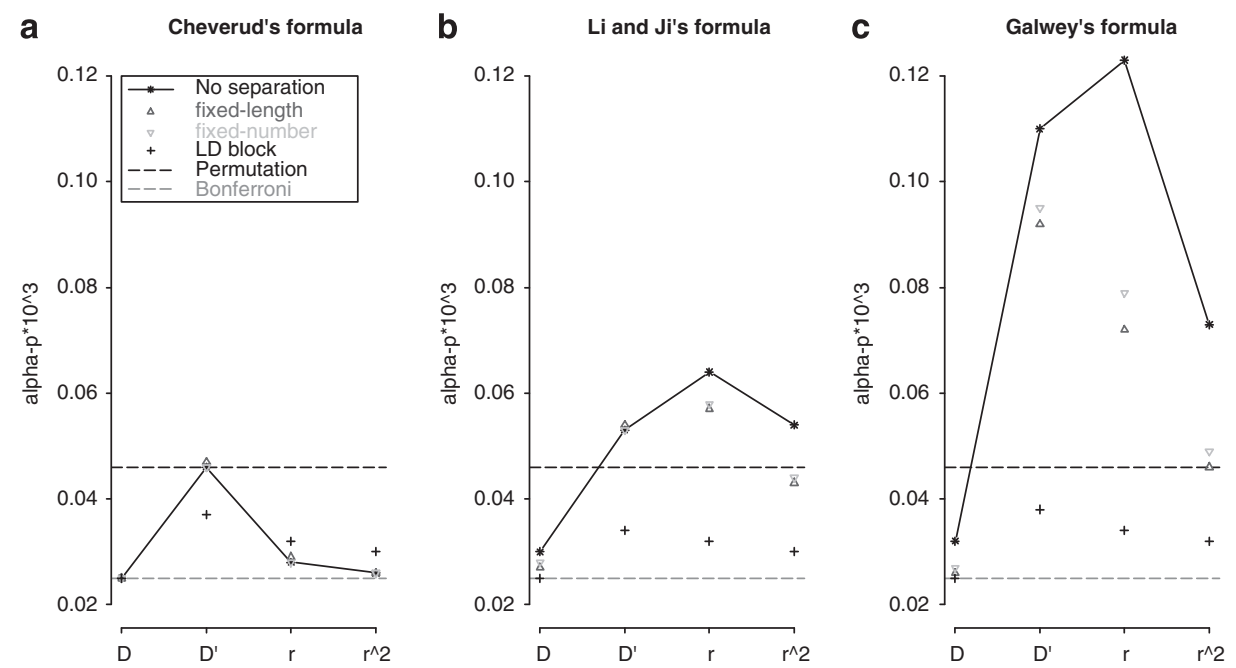

Figure 3 Significance thresholds from three principal component analysis (PCA)-based formulas, the permutation test and Bonferroni's correction for 2000 single nucleotide polymorphisms (SNPs) $(8588 \mathrm{~kb}$ ) by different separation methods. (a) Cheverud's formula; (b) Li and Ji's formula; (c) Galwey's formula. Black solid line denotes the overall analysis without separation, heavy blue-filled triangle point-up denotes fixed-length separation, light blue triangle pointdown denotes fixed-number separation and black cross denotes linkage disequilibrium (LD) block. Black dotted line represents the permutation test and pink dotted line denotes Bonferroni's correction. A full color version of this figure is available at the Journal of Human Genetics journal online.

YRI; as expected, the LD of the African population was weaker. Compare YRI with Asian and European population, the permutation test can reflect that the magnitude of LD becomes weaker; however, the analysis results found a small decline in $\alpha_{p}$ based on PCA-based formulas. This finding indicated that these three formulas would not be sensitive to detect differences in the strength of correlation among $M$ SNPs. As shown in Figure 3, the accuracy of PCA-based formulas changed in relation to the study populations. For Asian populations, $\mathrm{Li}$ and Ji's formula with either $D^{\prime}$ or $r^{2}$ coefficient obtained similar threshold as in the permutation test; however, it might be overestimated for CEU and YRI.

\section{The influences of large $M$}

The computation complexity of obtaining eigenvalues from the $M \times M$ LD matrix would not be efficient, if applied to numerous markers; for example, where $M$ is larger than 5000 . Therefore, we proposed three separation methods for the large $M$. The first separation method was fixed length, and we used $500 \mathrm{~kb}$ apart as a subset. For example, we selected 2000 SNPs $(8588 \mathrm{~kb})$; there were 18 subsets with 111 SNPs on average per subset (range: $21-156 \mathrm{SNP}$ ). The second dividing method was fixed-number separation; we set 200 markers as a group, and a total of 10 sets with $792 \mathrm{~kb}$ on average were obtained. The third one 'LD block' was to find the haplotype block by Haploview software ${ }^{31}$ (version 4.1) with Gabriel et al. ${ }^{32}$ definition of haplotype blocks. There were 1226 SNPs that fell within either 293 blocks; another 774 SNPs not located within the blocks were viewed as the independent tests. The range for SNPs within block is between 2 and 24 SNP. Hence, the total effective number can be written as:

$$
M_{\mathrm{eff}}=\sum_{i=1}^{B} M_{\mathrm{eff}(\mathrm{i})}+\sum_{j=1}^{M}\left(1-k_{\mathrm{j}}\right), k_{\mathrm{j}}=I\left(\text { snp }_{\mathrm{j}} \text { falls within block }\right)
$$

where $\mathrm{B}=293$ is the total number of subsets and $k$ is an indicator function for the j-th SNP that falls within block.

The adjusted significance level profiles from three separation methods are shown in Figure 3. The permutation test for the entire 2000 SNPs was viewed as the standard $\left(\alpha_{\mathrm{p}-\text { permut }}=4.6 \times 10^{-5}\right)$.
Compared with the permutation test and the Bonferroni's method $\left(\alpha_{\mathrm{p}-\text { Bonf }}=2.5 \times 10^{-5}\right)$, the patterns among the three formulas are very similar under the fixed-length and fixed-number separation, and close to that from the overall analysis without separation of 2000 SNPs. Although the separation methods lead to overall declining thresholds for Galwey's formula, the thresholds are still too liberal based on $D^{\prime}$ and $r$ coefficient. We found that Cheverud's formula based on $D^{\prime}$ coefficient performs as well as the permutation test, as well as Li \& Ji's and Galwey's formula with $r^{2}$ coefficient. Moreover, for separation methods, the required computation time is at most $0.35 \mathrm{~h}$ on a PC (Intel (Santa Clara, CA, USA) Core 2 Quad Q8200/2.33 GHZ CPU with $2 \mathrm{~GB}$ memory), and it is more time efficient than the overall analysis of 2000 SNPs based on each formula $(3.91 \mathrm{~h})$ and permutation test $(8.51 \mathrm{~h})$. However, the LD block leads to a more conservative threshold for each of the three formulas. One explanation for this is that the sample size (65 controls) was not enough for constructing reliable haplotype blocks ${ }^{33}$ as these blocks are relatively small with few SNPs. In other words, the conservativeness might be because of a large portion of SNPs $(774 / 2000=38.7 \%)$ that did not fall within either 293 blocks.

To examine the performance of fixed-length and fixed-number separation methods, we first use all SNPs on chromosome 10 to perform the estimation of effective numbers (Figure 4 shows the results for the fixed-length separation and Supplementary Figure S4 represents the fixed-number separation). Take Figure 4a for illustration, the estimated threshold of 22144 SNPs from the permutation test is $\alpha_{\mathrm{p} \text {-permut }}=4.3 \times 10^{-6}$ and comparable to that occurring on Chromosome 10 for the Affymetrix $500 \mathrm{~K}$ data in Table II from Gao et al.; ${ }^{34}$ however, the computation time is $96.12 \mathrm{~h}$. Cheverud's formula with $D^{\prime}$ coefficient for either fixed-length separation based on $500 \mathrm{~kb}$ or fixed-number separation based on 200 SNPs still obtained a reliable result similar to that obtained in the permutation test. Also, Li \& Ji's formula with $r^{2}$ coefficient performs as well as the standard. Furthermore, the separation methods did save much time $(5.49 \mathrm{~h})$ and were more efficient than the permutation test. For different population samples from the HapMap project (Figures $4 \mathrm{~b}-\mathrm{f}$ ), the results of 42610 commonly shared SNPs based on adequate separation methods 
a

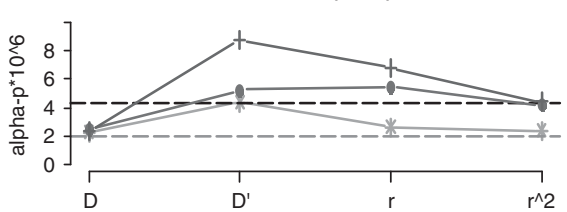

C
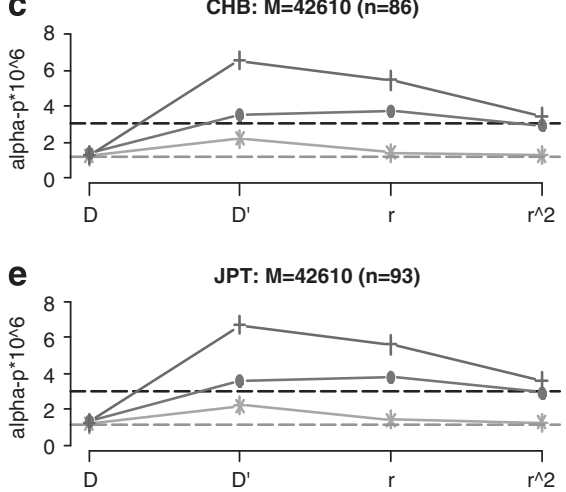

b $\quad$ CEU: $M=42610(n=174)$
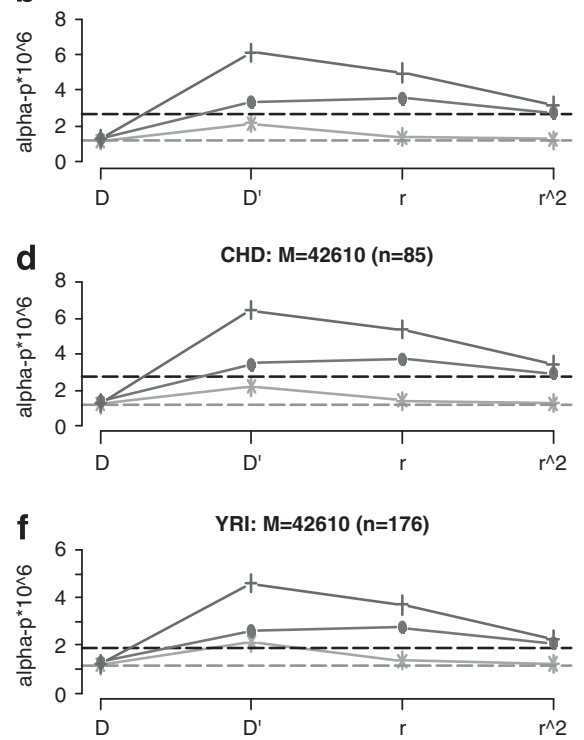

Figure 4 Significance thresholds from three principal component analysis (PCA)-based formulas with fixed-length separation, the permutation test and Bonferroni's correction in different populations using all single nucleotide polymorphisms (SNPs) on chromosome 10. (a) Data from Sarasquete et al.; ${ }^{8}$ (b) CEU; (c) CHB; (d) CHD; (e) JPT; and (f) YRI from the HapMap data. Green line denotes the Cheverud's formula, red line denotes Li and Ji's formula and blue line denotes Galwey's formula. Black dotted line represents the permutation test and pink dotted line denotes Bonferroni's correction. A full color version of this figure is available at the Journal of Human Genetics journal online.
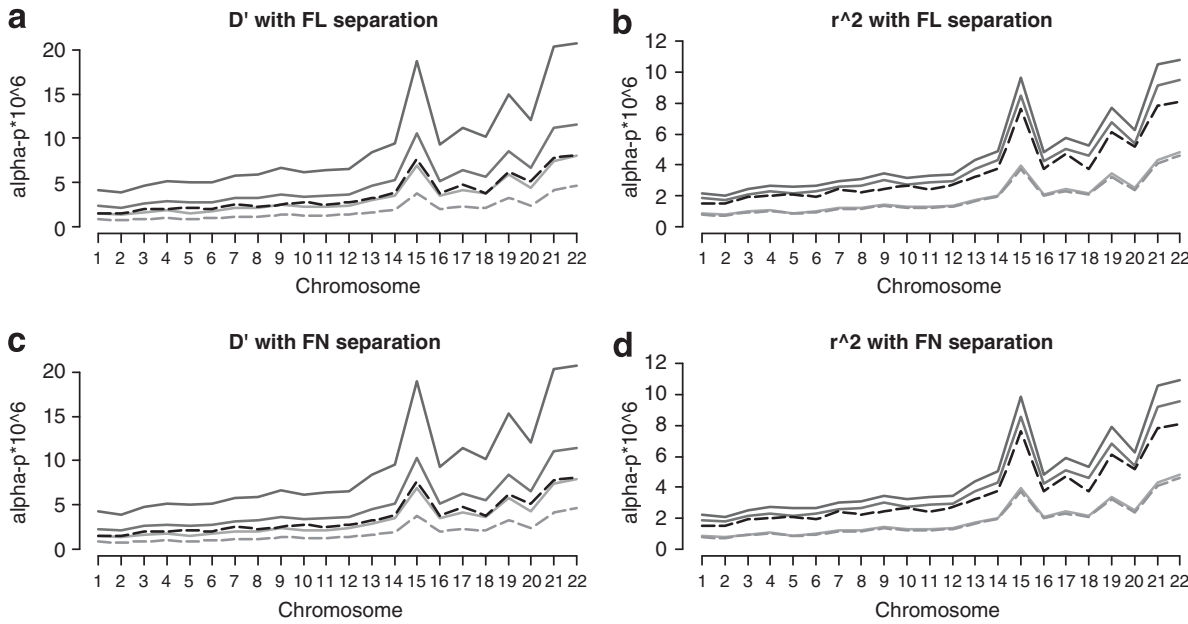

Figure 5 Significance thresholds from three principal component analysis (PCA)-based formulas based on separation methods, the permutation test and Bonferroni's correction using the CEU data from the HapMap project (810126 single nucleotide polymorphisms (SNPs) for 22 chromosomes). (a) $D^{\prime}$ coefficient with fixed-length separation; (b) $r^{2}$ coefficient with fixed-length separation; (c) $D^{\prime}$ coefficient with fixed-number separation; and (d) $r^{2}$ coefficient with fixed-number separation. Green line denotes the Cheverud's formula, red line denotes Li and Ji's formula and blue line denotes Galwey's formula. Black dotted line represents the permutation test and pink dotted line denotes Bonferroni's correction. A full color version of this figure is available at the Journal of Human Genetics journal online.

(that is, fixed-length separation based on $500 \mathrm{~kb}$ or fixed-number separation based on 200 SNPs) are consistent to those found for a small scale of SNPs (that is, 2000) without separation. In other words, Li \& Ji's formula with $r^{2}$ coefficient performs very close to the standard for Asian population; however, it might be somewhat liberal for CEU and YRI. It is noteworthy mentioning that Galwey's formula with $r^{2}$ coefficient based on separation methods obtains much closer results to the permutation test than that without separation.

Next, we evaluate the three PCA-based formulas using the CEU sample $(n=174)$ on a genome-wide scale either based on fixed-length (that is, $500 \mathrm{~kb}$ ) or fixed-number (that is, $200 \mathrm{SNPs}$ ) separation.
In total, 810126 SNPs with MAF > 5\% over 22 chromosomes were analyzed. We calculate the effective number for each chromosome according to the two separation methods. To demonstrate the computational time, we use chromosome 2 for an example. There were 68675 SNPs in chromosome 2. Calculating the effective number with fixed-length separation, it took about $17 \mathrm{~h}$ for a total of 478 sets. When we used the fixed-number separation, it took about $14.4 \mathrm{~h}$ for a total of 344 sets. In comparison, the permutation test would take more than 10 days on a PC. To save time, we used a workstation with 16 cores Intel Xeon E5520 cpus for permutation test with 10000 replications on genome-wide data for CEU. We also implement the fisher's exact 
test for the single marker-association test in parallel $\mathrm{R}$ programming on the workstation. However, it still took more time (about $41 \mathrm{~h}$ ) on a workstation than that for deriving the effective number on a single PC. The results for each chromosome are listed in Supplementary Table S2. For each chromosome, we saw a similar pattern as Figure $4 \mathrm{~b}$. Figure 5 shows the genome-wide pattern for the three PCA-based methods with $D^{\prime}$ and $r^{2}$ coefficient. Through adequate separation methods of large $M$, Cheverud's formula with $D^{\prime}$ coefficient would perform closer to the permutation test. In addition, $\mathrm{Li}$ and Ji's formula with $r^{2}$ coefficient obtain the similar threshold as the standard.

To validate the accuracy of the permutation test, we tried several replication times for the CYP2D6 data and found that the $\alpha_{\text {p-permut }}=0.00239$ from 10000 times was very similar to those from larger replications $\left(\alpha_{\mathrm{p}-\mathrm{permut}}=0.00238\right.$ at 20000 times, and $\alpha_{\mathrm{p}-\text { permut }}=0.00232$ at 50000 times). Furthermore, it is known that the distribution of minimum $P$-values from the permutation test should follow a Beta distribution with parameters $\left(1, M_{\text {eff }}\right)$, if there really exists an effective number $M_{\text {eff. Therefore, we fitted the Beta }}$ $(\mathrm{a}, \mathrm{b})$ distribution to the minimum $P$-values of the permutation tests for each scenario. The two parameters $(\mathrm{a}, \mathrm{b})$ were estimated by the maximum likelihood estimate (MLE) using SAS 9.1.3 (SAS Institute, Cary, NC, USA), with the first parameter set to 1 and with both parameters free. Supplementary Table S3 shows the estimated two Beta parameters for the CYP2D6 data $^{28}$ and the chromosome 10 data. ${ }^{8}$ Without setting $\mathrm{a}=1$, we found that the estimate $\hat{a}$ was close to 1 for each case. In addition, the estimate $\hat{b}$ was in line with the effective number obtained by the permutation tests corresponding to either $\mathrm{a}=1$ or a was free. Thus, the threshold with 10000 replication times was reliable.

\section{DISCUSSION}

We have examined the performance of three common PCA-based formulas separately proposed by Cheverud ${ }^{16}, \mathrm{Li}$ and $\mathrm{Ji}^{18}$ and Galwey ${ }^{20}$ for deriving the effective number of tests in GAS. Considering the influences of LD measurements, the estimated effective numbers according to three formulas are indeed very different. The estimated effective numbers are more strongly affected by $D^{\prime}$ and $r$ coefficient than those with Lewontin's $D$ coefficient. Excluding $D$ coefficient, the magnitudes of the effective numbers from Galwey's formula are the smallest (that is, the largest $\alpha_{\mathrm{p}}$ ), followed by Li and Ji's, and the largest for Cheverud's formula. Similar patterns were also observed with application to the HapMap data consisting of various population samples. Although Cheverud's formula was previously proved accurately, but at the current large scale of $M$, adopting the variance of $M$ eigenvalues would not be able to well capture the simultaneous correlations among $M$ SNPs. In general, the estimated effective numbers from Li and Ji's formula were relatively more stable than those from the other two formulas. Currently, the total number of markers in GAS is tremendous, and the SNP density is expected to be small. As for the impact of marker density, Cheverud's formula could not reflect the differences under different marker densities constrained by the same markers. Li and Ji's formula and Galwey's formula with the application to $r^{2}$ coefficient highlight differences as compared with other LD coefficients. Compared with the Bonferroni's method and permutation test, Cheverud's formula is the most stringent, followed by Li and Ji's, and Galwey's formula is more liberal. Furthermore, we found that $\mathrm{Li}$ and Ji's formula with the application to $r^{2}$ coefficient obtained the best estimation most frequently. Additionally, Cheverud's formula with $D^{\prime}$ coefficient would perform the best under smaller marker density ( $5 \mathrm{~kb}$ per SNP) and small sample size. In other words, Cheverud's formula could take advantage of the overestimation of $D^{\prime}$ coefficient and improve the over conservativeness of $M_{\mathrm{eff}-\mathrm{C}}$ under small sample size. For controlling the overall type I error rate adequately and properly, Li and Ji's formula with $r^{2}$ coefficient is recommended, as $\mathrm{Li}$ and Ji's formula can support additional factors including the marker density, the total number of markers and separation methods. Besides, it is more stable compared with Galwey's formula, although Galwey's formula with $r^{2}$ coefficient obtains similar threshold as the permutation test in some cases. Third, $\mathrm{Li}$ and Ji's formula makes an improvement in the eigenvalue-greater-than-one rule $^{18}$ for calculating the effective number, as well as the $r^{2}$ coefficient does not suffer as much from small sample size problem as $D^{\prime}$ coefficient. ${ }^{35}$

With the increasing genome-wide association studies, the effective number approaches can serve as a fast approximation to the permutation test in terms of time efficiency. However, the limitation would be the computational burden with a large number of SNPs. For effective number of independent test based on the PCA, it would not be feasible to complete the matrix decomposition over hundreds of thousands of SNPs. Hence, adequate separation methods would make it applicable to the genome-wide scale. We have shown that the division methods of SNPs based on the fixed-length (that is, $500 \mathrm{~kb}$ ) and fixed-number (that is, 200 SNPs) separation did have more accurate estimates and save more time. In particular, the accuracy of three formulas did improve better through adequate separations over a large number of SNPs, especially for the Galwey's formula with $r^{2}$ coefficient. Although Cheverud's formula with $D^{\prime}$ coefficient could obtain the most accurate result, the cases we considered had a small sample size $(n=174)$. Taking into account the weakness of the inflation of $D^{\prime}$ coefficient by small sample size, we recommend using $\mathrm{Li}$ and Ji's formula with $r^{2}$ coefficient for the correlated multiple testing in large-scale GAS. For a valid examination of LD blocks separation, further study will be required to use data with hundreds of samples as suggested in Osabe et al..$^{33}$ There may be some issues for the PCA-based formulas in calculating the effective number. One problem with PCA is the missing data in SNP genotypes. For example, the composite LD proposed in Gao et al. ${ }^{29}$ could be obtained only from the complete data. Missing genotypes can be filled with new developments in genotyping technology and/or statistical imputation methods. However, a small number of missing values will be unlikely to hinder the inference of the PCA-based formulas. Hence, further study is needed to examine the influences of other types of LD measurements such as the statistical correlation coefficients and composite LD obtained from the complete data. Another issue relates to the sample size. We have considered large-scale association studies with moderate sample size (65-176), but we should expect similar patterns of three PCAbased formulas as would be applied to a larger sample size (refer to the results of CYP2D6 data, although $M=27$ is small). However, the performance of good approximation with the standard (that is, the permutation test) would likely alter according to different population samples. For example, we found that $\mathrm{Li}$ and Ji's formula with $r^{2}$ coefficient performs better for Asian populations, but it is somewhat liberal for the CEU and YRI population. The results in this study can provide practical guidelines for calculating an effective number and be useful to current large-scale GAS.

\section{ACKNOWLEDGEMENTS}

We would like to acknowledge Louise Hosking, from GlaxoSmithKline, for providing the CYP2D6 data set. We are grateful to Hong-Chun Hsu and Wen-Cheng Lin for providing the workstation PC and technical support, and Ying-Lu Li and You-Syuan Li for assisting in selecting the commonly shared SNPs on 22 chromosomes of the HapMap data and to the reviewers for their helpful comments. This work has been partially supported by 
Grants NSC97-2118-M-320-001-MY2 from the Taiwan National Science Council.

1 International SNP Map Working Group. A map of human genome sequence variation containing 1.42 million single nucleotide polymorphism. Nature. 409, 928-933 (2001).

2 Olivier, M. A haplotype map of the human genome. Physiol. Genomics. 13, 3-9 (2003).

3 The International HapMap Consortium. A second generation human haplotype map of over 3.1 million SNPs. Nature. 449, 851-861 (2007).

4 Hirschhorn, J. N. \& Daly, M. J. Genome-wide association studies for common diseases and complex traits. Nat. Rev. Genet. 6, 95-108 (2005).

5 Hunter, D. J., Kraft, P., Jacobs, K. B., Cox, D. G., Yeager, M., Hankinson, S E. et al. A genome-wide association study identifies alleles in FGFR2 associated with risk of sporadic postmenopausal breast cancer. Nature. 39, 870-874 (2007).

6 Klein, R. J., Zeiss, C., Chew, E. Y., Tsai, J. Y., Sackler, R S., Haynes, C. et al. Complement factor $\mathrm{H}$ polymorphism in Age-Related Macular Degeneration. Science. 308, 385-389 (2005).

7 Van Es, M. A., Van Vught, P. W., Blauw, H. M., Franke, L., Saris, C., Anderson, P. et al. ITPR2 as a susceptibility gene in sporadic amyotrophic lateral sclerosis: a genome-wide association study. Lancet. Neurology. 6, 869-877 (2007).

8 Sarasquete, M., Garcia-Sanz, R., Marin, L., Alcoceba, M., Chillon, M., Balanzategui, A. et al. Bisphosphonate-related osteonecrosis of the jaw is associated with polymorphisms of the cytochrome P450 CYP2C8 in multiple myeloma: a genome-wide single nucleotide polymorphism analysis. Blood. 112, 2709-2712 (2008).

9 Tanaka, Y., Nishida, N., Sugiyama, M., Kurosaki, M., Matsuura, K., Sakamoto, N. et al. Genome-wide association of IL28B with response to pegylated interferon- $\alpha$ and ribavirin therapy for chronic hepatitis C. Nat. Genet. 41, 1105-1109 (2009).

10 Risch, N. \& Merikangas, K. The future of genetic studies of complex human diseases. Science. 273, 1516-1517 (1996).

11 Thomas, D. C., Haile, R. W. \& Duggan, D. Recent developments in genome-wide association scans: a workshop summary and review. Am. J. Hum. Genet. 77, 337-345 (2005).

12 Balding, D. J. A tutorial on statistical methods for population association studies. Nat. Rev. Genet. 7, 781-791 (2006).

13 Dudoit, S., Shaffer, J. P. \& Boldrick, J. C. Multiple hypothesis testing in microarray experiments. Stat. Sci. 18, 71-103 (2003).

14 Botstein, D. \& Risch, N. Discovering genotypes underlying human phenotypes: past successes for mendelian disease, future approaches for complex disease. Nat. Genet. 33, 228-237 (2003).

15 Churchill, G. \& Doerge, R. Empirical threshold values for quantitative trait mapping. Genetics. 138, 963-970 (1994).

16 Cheverud, J. M. A simple correction for multiple comparisons in interval mapping genome scans. Heredity. 87, 52-58 (2001).
17 Nyholt, D. R. A simple correction for multiple testing for single-nucleotide polymorphisms in linkage disequilibrium with each other. Am. J. Hum. Genet. 74, 765-769 (2004).

$18 \mathrm{Li}, \mathrm{J} . \& \mathrm{Ji}$, L. Adjusting multiple testing in multilocus analyses using the eigenvalues of a correlation matrix. Heredity. 95, 221-227 (2005).

19 Kaiser, H. F. The application of electronic computers to factor analysis. Educ. Psychol. Meas. 20, 141-151 (1960).

20 Galwey, N. W. A new measure of the effective number of tests, a practical tool for comparing families of non-independent significance tests. Genet. Epidemiol. 33, 559-568 (2009).

21 Patterson, N., Price, A. L. \& Reich, D. Population structure and eigenanalysis. PLoS Genet. 2, e190 (2006).

22 Dudbridge, F. \& Gusnanto, A. Estimation of significance thresholds for genomewide association scans. Genet. Epidemiol. 32, 227-234 (2008).

23 Lewontin, R. C. The interaction of selection and linkage. I. General considerations: heterotic models. Genetics. 49, 49-67 (1964).

24 Hill, W. G. \& Robertson, A. Linkage disequilibrium in finite populations. Theor. Appl. Genet. 38, 226-231 (1968).

25 Devlin, B. \& Risch, N. A comparison of linkage disequilibrium measures for fine-scale mapping. Genomics. 29, 311-322 (1995).

26 Pritchard, J. \& Przeworski, M. Linkage disequilibrium in humans: models and data. Am. J. Hum. Genet. 69, 1-14 (2001).

27 Conrad, D. F., Jakobsson, M., Coop, G., Wen, X., Wall, J. D., Rosenberg, N. A. et al. A worldwide survey of haplotype variation and linkage disequilibrium in the human genome. Nat. Genet. 38, 1251-1260 (2006)

28 Hosking, L., Boyd, P., Xu, C., Nissum, M., Cantone, K., Purvis, I. et al. Linkage disequilibrium mapping identifies a $390 \mathrm{~kb}$ region associated with CYP2D6 poor drug metabolising activity. Pharmacogenomics. J. 2, 165-175 (2002).

29 Gao, X., Starmer, J. \& Martin, E. A multiple testing correction method for genetic association studies using correlated single nucleotide polymorphisms. Genet. Epidemiol. 32, 361-369 (2008).

30 R Development Core Team. R: A Language and Environment for Statistical Computing (Vienna, Austria: R Foundation for Statistical Computing) (2008).

31 Barrett, J. C., Fry, B., Maller, J. \& Daly, M. J. Haploview: analysis and visualization of LD and haplotype maps. Bioinformatics. 21, 263-265 (2005).

32 Gabriel, S. B., Schaffner, S. F., Nguyen, H., Moore, J. M., Roy, J., Blumenstiel, B. et al. The structure of haplotype blocks in the human genome. Science. 296, 2225-2229 (2002).

33 Osabe, D., Tanahashi, T., Nomura, K., Shinohara, S., Nakamura, N., Yoshikawa, T. et al. Evaluation of sample size effect on the identification of haplotype blocks. BMC Bioinformatics. 8, 200 (2007).

34 Gao, X., Becker, L. C., Becker, D. M., Starmer, J.D. \& Province, M.A. Avoiding the high Bonferroni penalty in genome-wide association studies. Genet. Epidemiol. 34, 100-110 (2010).

35 Ardlie, K. G., Kruglyak, L. \& Seielstad, M. Patterns of linkage diseqquilibrium in the human genome. Nat. Rev. Genet. 3, 299-309 (2002).

Supplementary Information accompanies the paper on Journal of Human Genetics website (http://www.nature.com/jhg) 